\title{
Automated location of fiber Bragg gratings in draw tower grating arrays using a thermal scanning technique
}

\author{
Simon Maunder, ${ }^{a, *}$ Neil Blenman, ${ }^{a}$ lan Underhill, ${ }^{b}$ John Arkwright, ${ }^{c}$ and Eric Lindnerd \\ ${ }^{a} C S I R O$ Materials Science and Engineering, Lindfield, NSW 2070, Australia \\ ${ }^{\mathrm{b}}$ Griffith University, Griffith School of Engineering, Gold Coast Campus, QLD 4222, Australia \\ ${ }^{c}$ Flinders University, School of Computer Science, Engineering and Mathematics, SA 5001, Australia \\ ${ }^{\mathrm{d}}$ FBGS Technologies GmbH, Winzerlaer Straße 2, Jena D-07745, Germany
}

\begin{abstract}
For some critical applications, the location of fiber Bragg gratings (FBGs) in draw tower grating (DTG) arrays needs to be determined to sub-mm accuracy; for example, successful packaging of the FBGs in $\mathrm{mm}$ scale packages. The DTG manufacturing process leaves no external visible identification marks on the fiber, hence location needs to be determined prior to packaging. This work presents an automated fiber marking system that can accurately locate the positions of the FBGs within $\pm 0.1 \mathrm{~mm}$. The simple, low cost, and automated system avoids manual fiber handling and allows accurate packaging of the FBGs for sensing applications. @ 2014 Society of Photo-Optical Instrumentation Engineers (SPIE) [DOI: 10.1117/1.OE.53.8.084113]
\end{abstract}

Keywords: fiber Bragg grating; fiber optics; draw tower; location; heating.

Paper 140974 received Jun. 19, 2014; revised manuscript received Jul. 23, 2014; accepted for publication Aug. 6, 2014; published online Aug. 26, 2014.

\section{Introduction}

Fiber Bragg gratings (FBGs) are one of the mainstays of optical fiber sensing and are now common in exploration and mining, infrastructure, structural health monitoring, and increasingly, in medical applications. The process for inscribing FBG elements into fiber is now universally done using an interferometric side writing technique, in which the fiber is directly exposed to an ultraviolet (UV) interference pattern. For UV inscription, this requires direct exposure of the glass surface and must be carried out without the normal protective fiber coating in place. This can be achieved either by "window-stripping" the fiber, i.e., removing a section of the protective coating in order to access the glass surface of the fiber, or by inscription before the coating is applied. In the former case, the window-stripping process invariably weakens the fiber ${ }^{1}$ and increases the risk of subsequent fiber breakage. In the latter case, the only viable way of doing this is by inscribing the FBGs during the fiber drawing process, after the fiber diameter has stabilized, but before the protective coating has been applied. This 'Draw-Tower Grating' (DTG) process ${ }^{2}$ results in significantly higher strength fibers than the window stripped approach and is ideal for sensing purposes in which the flexibility of the fiber and exposure of the FBG region to ambient environments are required.

However, the DTG process results in a uniformly coated fiber with no visible indication of the locations of the inscribed FBG structures. The DTG arrays supplied by FBGS $^{3}$ contain marks approximately $15 \mathrm{~mm}$ from each end of the DTG array, but no accurate markings to identify the precise location of each FBG element. This may be suitable for some applications, but we require sub $\mathrm{mm}$ to accurately package the FBGs within the array. A further issue is that cleaning the fiber in preparation for epoxy bonding will also remove any identification marks along the fiber, requiring the checking and marking of each FBG location before assembly into precision transducer housings as is needed for the high resolution manometry (HRM) pressure sensor catheters recently developed by our group. ${ }^{4,5}$ This again leads to the problem of accurately locating the FBG position within the optical fiber after the draw.

Failure to accurately position each FBG structure within the sensor substrate can result in disruption of the spectral profile of the grating due to the additional strain generated in the fiber by the epoxy joint. Figure 1 shows the spectral profiles of an FBG correctly bonded into one of our HRM transducers and one that is partially located within the epoxy region. These two different regions of the FBG (bonded and unbonded) result in two separate peaks that render the device inoperable. This is compounded by the DTG arrays used, which contain up to 36 spectrally separated FBGs on each fiber, all requiring accurate placement in their respective substrates.

Methods that have been reported for noncontact FBG locating and characterization have included various side scanning techniques. ${ }^{6,7}$ However, they can only be achieved on stripped fiber and, therefore, are not suitable for use on the DTG arrays. They can also require precise optical alignment or the application of an index matching liquid prior to the measurement. Heat scanning techniques have also been used to characterize FBGs that induce a phase change in the FBG spectra. ${ }^{8-10}$ Limitations include slow scanning times to allow for thermal stabilization, requiring up to 20 min for FBG characterization. These combined limitations of scanning time, setup complexity, stripping coating, and resolution meant they were not directly suitable for the 


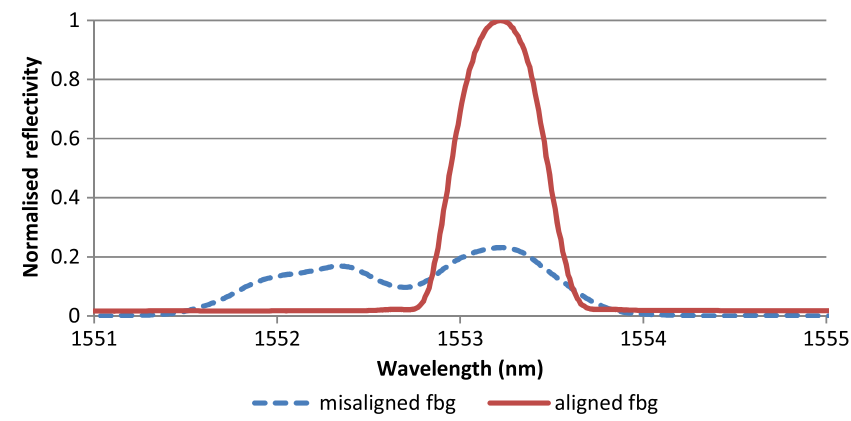

Fig. 1 Example of FBG spectra misaligned in sensor substrate, showing a double peak due to a single grating being exposed to different environments within the catheter pressure sensor compared to the aligned FBG spectra in the sensing substrate.

basis for the automated marking of long DTG arrays as part of a device manufacturing process.

\section{Automated Fiber Marking System}

The automated FBG marking system (Fig. 2) was developed to find the locations of all gratings in an array within $\pm 0.1 \mathrm{~mm}$, and then place an ink mark at each FBG location on the fiber. This is necessary to avoid misaligned FBGs in transducer packages, which can result in a perturbation of the FBG spectrum and inaccuracies in location of the spectral peak. Using this apparatus, the DTG arrays can be thoroughly cleaned in preparation for epoxy bonding, and then loaded into the jig, marked, and unloaded without subsequent handling of the fiber. This level of cleanliness proved essential for good quality epoxy bonding of the fiber to the substrates. The process also provided a visual reference mark located at the center of each FBG structure for alignment to its respective transducer housing, avoiding any potential misalignment of the FBGs.

The sensitivity of the fiber marking system was such that it showed a very small oscillation in the pitch between the
FBGs. The calculated oscillation equates to a capstan circumference of $70 \mathrm{~mm}$ and shows the eccentricity in the capstan from the fiber draw tower that causes a pitch oscillation of $0.2 \mathrm{~mm}$. This small variation in pitch did not present any issues with the DTG arrays or with subsequently fabricated catheters; however, it nicely indicates the sensitivity and repeatability of the marking jig.

The fiber marking system we have developed is based on the noncontact heat scanning technique. When used on short length DTG arrays with 3-mm long FBGs and where the fiber remains coated, it can provide a simple setup with faster scan times and high accuracy when only the FBG location is required. This method will work for FBGs of different lengths as long as the hot wire generates a uniform shift in the reflected spectral peak and not a localized change in optical period, which would, in turn, generate a phaseshifted spectrum that could confuse the peak finding algorithm. The system is a low cost and simple design with a stepper motor and lead screw driving the hot wire and print head carriage assembly at a constant speed during FBG scanning. The resistance hot wire is set up on an insulated block perpendicular to and within $1 \mathrm{~mm}$ under the fiber with a temperature of approximately $60^{\circ} \mathrm{C}$. Only one axis of movement is required along the fiber axis. The hot wire scans the DTG array to locate the position of each FBG within the array. The presence of a grating is detected by the thermally induced shift in wavelength generated as the hot wire passes underneath the grating. When a grating is detected, the carriage assembly switches to a search mode and moves back and forth under the grating an even number of times to accurately determine the center of the grating. An identification mark using an inkjet print module (Evolution 1) ${ }^{11}$ is applied, hence the accurate position can be visually identified and the pitch of the array is also calculated. The system is housed in an acrylic enclosure to reduce any external thermal influence on the fiber. The fiber is held suspended in position with pneumatic clamps against guide stops to allow rapid,

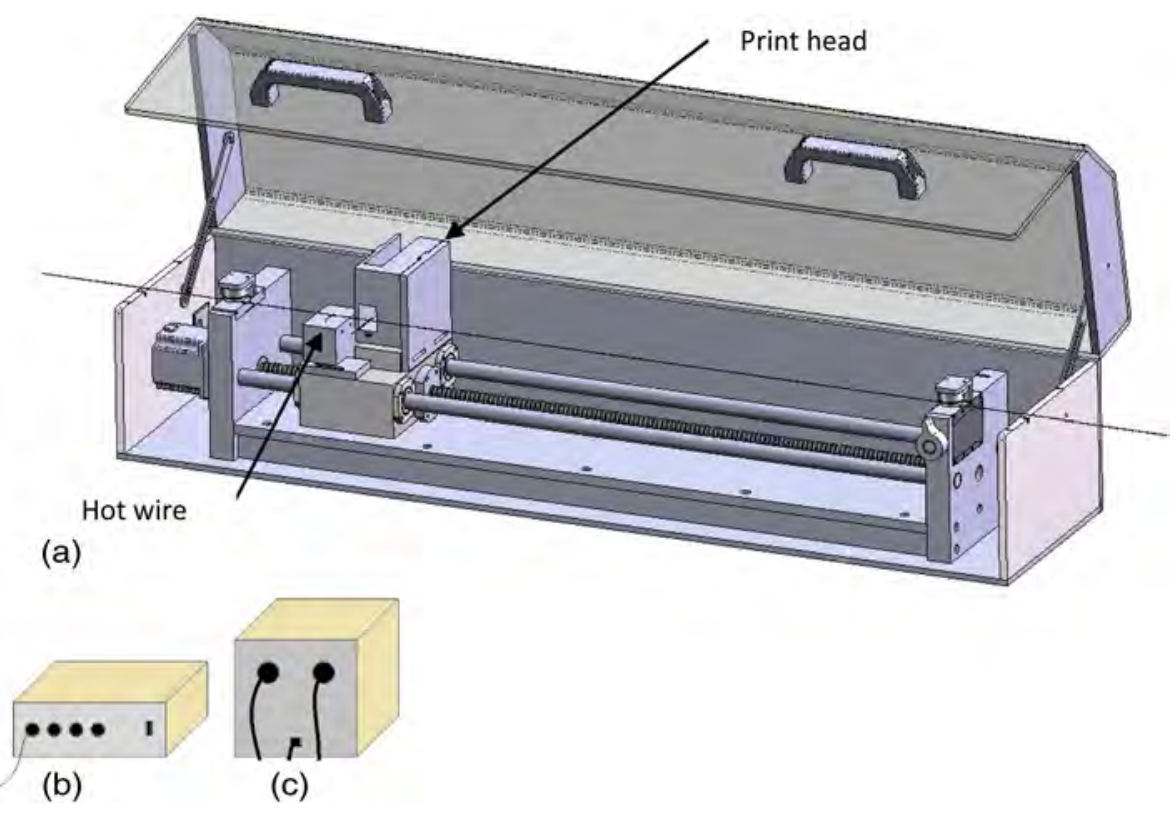

Fig. 2 Fiber marking system comprising a stepper motor driving a lead screw and carriage assembly with hot wire and print head, mounted in an enclosure (a), optical interrogator (b) and fiber marking control unit (c). 
accurate alignment of the fiber, and is held under slight tension to maintain linearity.

The system is PC software controlled using custom written LabView code (National Instruments, Austin), ${ }^{12}$ monitors all peak wavelengths with a commercial spectral interrogator unit (FOS\&S 702, FBGS Technologies GmbH, Jena, Germany) ${ }^{3}$ within a spectral range of $1515 \mathrm{~nm}$ to $1590 \mathrm{~nm}$, and can detect a FBG peak to a resolution of $1 \mathrm{pm}$. With an alternative spectral interrogator (BlueBox from IPHT Jena, Germany), ${ }^{13}$ FBGs from 805 to $855 \mathrm{~nm}$ have also been located and marked. The gratings can be in any arbitrary spectral order and physical spacing within the jig scanning range of $600 \mathrm{~mm}$ because of the interrogator's ability to selectively monitor individual FBGs. It can also locate multiple FBGs without having to reposition the fiber in the system.

\section{Characterization}

The software initially determines the number of FBGs present on the fiber by counting the number of spectral peaks detected. The hot wire stage then scans at a relative high speed of $2 \mathrm{~mm} / \mathrm{s}$ to approximately locate the first grating in the array by looking for a wavelength shift in any of the spectral peaks due to the temperature change. Note that this approach allows the system to locate the gratings in any spectral order rather than relying on a monotonic increase or decrease in spectral peaks. Once located, a detailed scan is performed at an optimum slower scanning rate of $1.1 \mathrm{~mm} / \mathrm{s}$ over a $10-\mathrm{mm}$ range, passing the grating in both directions to eliminate the thermal lag effects of the hot wire system (Fig. 3). The wavelength information is recorded by the spectral interrogator unit as a function of position for both directions and is used to calculate the center position of the FBG. For an equal number of scans, the positional variation is less than $10 \mu \mathrm{m}$ after only 2 scans, hence achieving the required accuracy in the shortest possible time. If a unidirectional scan is used, the calculated position is affected by thermal lag and can be out of position by up to $0.75 \mathrm{~mm}$ (Fig. 4). The FBG center position is calculated by the LabView peak wavelength algorithm and the position is then marked with a $0.3 \mathrm{~mm}$ width ink mark on the center of the FBG. Once located and marked, the process is then repeated for the next FBG along the fiber. Scan times for measuring and marking each single DTG are approximately $30 \mathrm{~s}$, and the

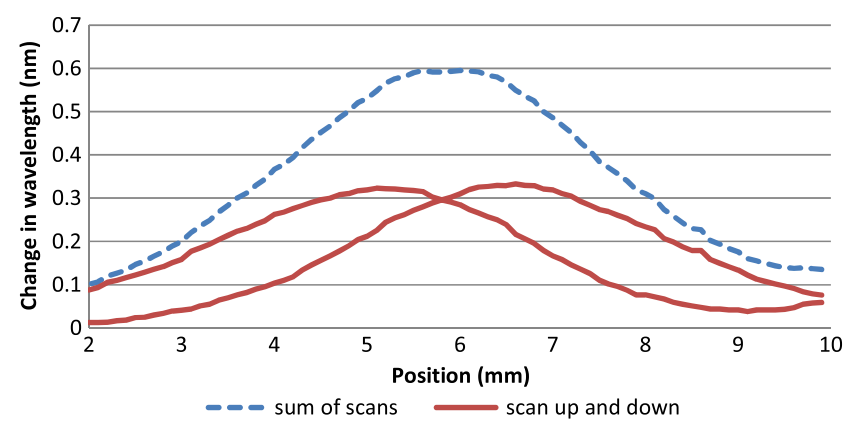

Fig. 3 Single FBG scan passing in the opposite direction showing the effect of thermal lag in the fiber as the hot wire system passes under the FBG that also shows an initial drop off from cooling from the pass in the opposite direction. The sum of scans in both directions produced an averaged scan used to calculate FBG position by using a peak find algorithm for the center position.

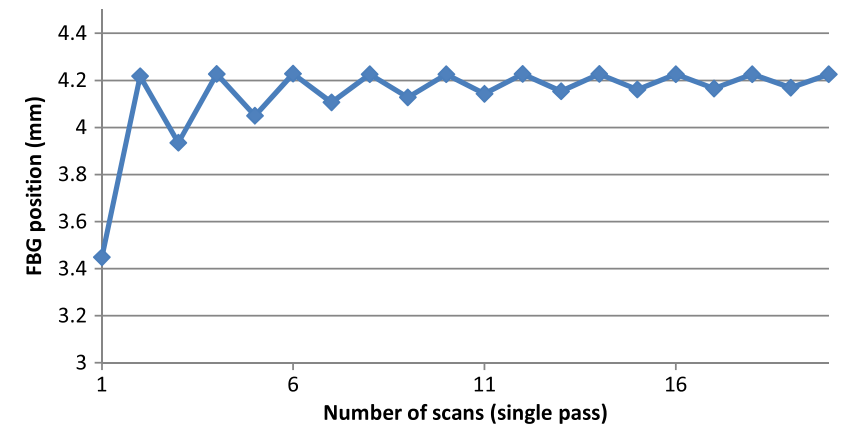

Fig. 4 FBG position as a function of the number of passes used in the calculation. An even number of scans equates to an equal number of passes in both directions, which cancel any hysteresis effects. In contrast, an odd number of scans creates errors of $>0.75 \mathrm{~mm}$ in the location.

process of translating for a full 36-element DTG array is on the order of $30 \mathrm{~min}$. This is limited in our prototype by the slow stepper motor controller system, but could easily be improved with a faster controller.

The relatively short length of the FBGs and the separation between the fiber and the hot wire means that the hot wire is effectively heating up the entire FBG, causing the grating to shift up in wavelength by approximately $0.3 \mathrm{~nm}$. The localized heat source does not create any spectral separation of the peak such as that seen in the previous heat scanning techniques, ${ }^{8-10}$ and allows fast continuous scanning with only peak wavelength detection algorithms. The low temperature and short exposure time do not create any annealing-induced changes in the grating profile or reflected peak wavelength. The ability to acquire an accurate location with such a small wavelength shift (Fig. 5) makes this method viable for closely spaced spectrally separated arrays. The minimum spectral separation between gratings is defined by the full width to the first zeroes of each grating and the thermal shift of the grating. In this instance, with gratings of $0.7 \mathrm{~nm}$ to the first zeroes and an induced spectral shift of $0.3 \mathrm{~nm}$, the lower limit for the spectral separation was $1 \mathrm{~nm}$. If a large wavelength shift is used, then it could not be used to find the FBG position because of the interference of the neighboring peak.

The fiber marking system has been shown to have an accuracy better than $0.1 \mathrm{~mm}$, and the very small cyclic pitch variations detected within the DTG arrays can be traced back to the variation in the capstan of the draw tower manufacturing process.

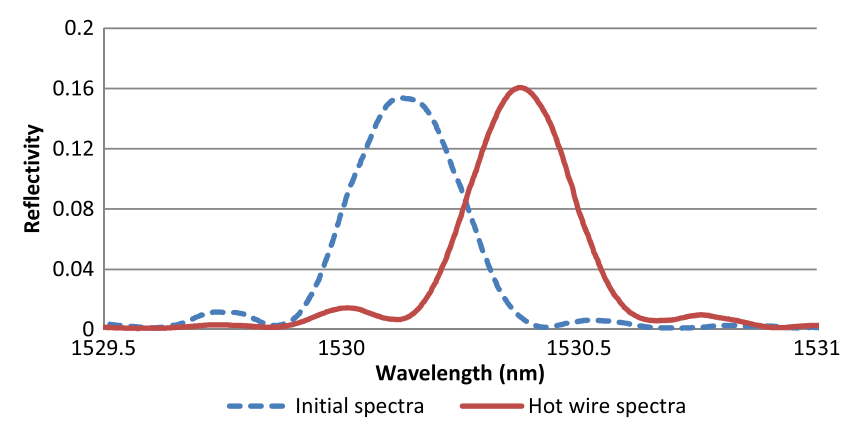

Fig. 5 Spectra observed before and during a hot wire pass showing a shift up in wavelength of the FBG. 


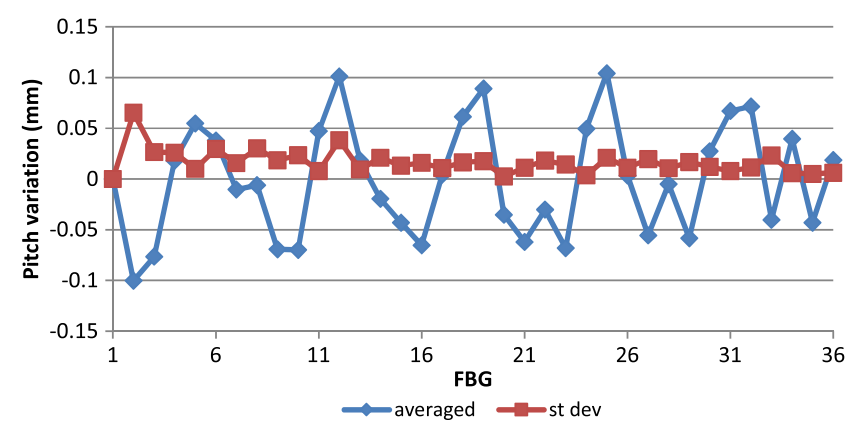

Fig. 6 Individual FBG array showing the scan pitch variation and the warm up start signature of the initial scan (standard deviation variation to left of chart).

\section{Results: Measuring Draw Tower Grating Arrays}

The DTG arrays used contain 36 FBGs at a nominal design pitch of $10.4 \mathrm{~mm}$. The length of the FBGs is $3 \mathrm{~mm}$ and the wavelength range is from a nominal 1517 to $1589 \mathrm{~nm}$.

Calculating the slope of the array gives the calculated pitch of the array at $10.44 \mathrm{~mm}$ and shows a very small periodicity in the pitch of the array over a nominal length of approximately 6.6 gratings which equate to approximately $68.9 \pm 1 \mathrm{~mm}$. Within the limits of the measurement, this closely matches the capstan circumference of $70 \mathrm{~mm}$ of the fiber draw tower used by FBGS. There is a slightly higher variation in the measured position at the start of the array due to a slight warm up signature generated by the stepper motor assembly, but this can be avoided if necessary by moving the array further away from the stepper motor assembly. The periodicity variation, although very small and well within the required specifications for the DTGs, remains clearly evident as shown in Fig. 6. It has been determined to be caused by a slight eccentricity in the capstan of the fiber draw tower. This causes an oscillation in the fiber draw speed during the manufacturing process, and thus gives the pitch variation observed on the DTG arrays.

Confirmation that the periodicity is in the DTG arrays and is not a measurement artifact due to the fiber marking system was performed by shifting a DTG array in the measurement system by half a period and remeasuring. Then the array is flipped again so it is measured in reverse orientation to eliminate any systemic or directional influences. Results show the periodicity variation is direction and position independent from the measurement system and is associated with the DTG array manufacturing process (Fig. 7).

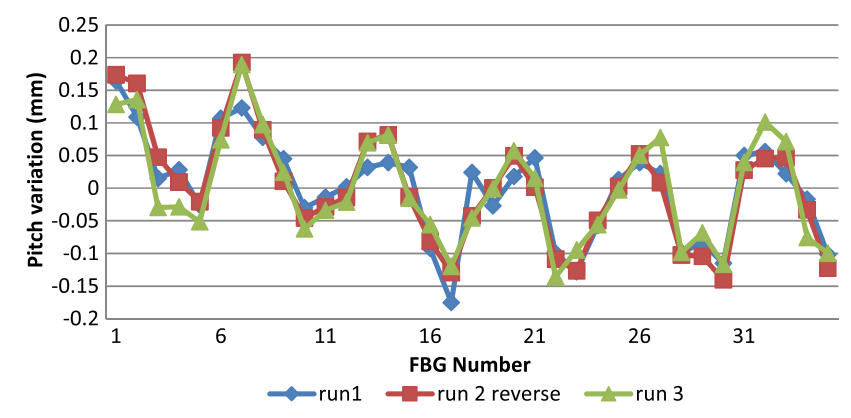

Fig. 7 Individual scans pitch variation compared to nominal pitch with reverse orientation and repositioning scans.

\section{Conclusion}

An automated noncontact fiber marking system was developed using a scanning hot wire. It is a simple low cost system, capable of recording wavelength as a function of position to calculate the location of spectrally separated FBGs. The system performs well enough to identify small periodic variations in the FBG pitch that are consistent and well within the desired accuracy for the array. It can automatically locate and mark the fiber at each FBG location, then calculate the average array pitch for subsequent accurate placement of the FBG on sensing substrates.

Limitations of the scanning time of the current system ( $\sim 30 \mathrm{~min}$ for a 36-element array) are due to the available stepper motor controller/PC combination. This could easily be improved by using a faster stepper motor controller, as well as by modifying the search routine to locate the FBGs first, then marking all the locations in a single run to reduce the overall travelled distance of the stage.

There is also a small warm up thermal effect due to the stepper motor on the fiber and system. This causes slightly larger variations in the detected pitch at the start of the measurement, but they are still within the design parameters. This should not affect the accurate placement of the ink marks on the fiber and remains within the $\pm 0.1 \mathrm{~mm}$ desired accuracy for the system. Using multiple scans in both directions for each FBG can yield an accuracy of $10 \mu \mathrm{m}$ for a single FBG, which is well within the overall positional error due to the finite size of the ink mark.

The advantage of the system is that it allows time saving through fiber handling by completing the whole array in one step without having to handle the clean fiber. This level of cleanliness is important for building our FBG-based sensing devices. The initial loading of the fiber array does not require accurate alignment in the system as the scan itself is selfaligning. The FBGs can also be in any order and spacing within the $600-\mathrm{mm}$ scanning range, providing a completely automated hands-free system to locate and mark FBG arrays. This is essential for DTGs, in which the fiber is coated after the grating is written, leaving no permanent visible marks on the fiber.

The small periodicity of $0.2 \mathrm{~mm}$ in the pitch of the detected DTG arrays has been determined to be a slight eccentricity in the capstan (circumference $70 \mathrm{~mm}$ ) of the fiber draw tower that occurred during the manufacturing process. This caused oscillations in the fiber draw speed during the manufacturing process. The manufacturing process, coupled with the constant laser pulse rate of the DTG writing process, gives the small pitch variation on the arrays that were able to be detected by the fiber marking jig.

\section{References}

1. T. WEI et al., "Degradation of fibre strength during coating stripping," in Proc. Int. Wire \& Cable Symp., pp. 199-204, US Army Communications-Electronics Command, Atlanta (1989).

2. M. W. Rothhardt, C. Chojetzki, and H. R. Mueller, "High-mechanicalstrength single-pulse draw tower gratings," Proc. SPIE 5579, 127-135 (2004).

3. FBGS Technologies GmbH, http://www.fbgs.com/ (18 6 2014).

4. J. W. Arkwright et al., "A fibre Bragg grating manometry catheter for in-vivo diagnostics of swallowing disorders," in Proc. OptoElectronics and Communications Conf. 2008, and the 2008 Australian Conf. on Optical Fibre Technology. OECC/ACOFT, pp. -, IEEE, Sydney, Australia (2008).

5. J. W. Arkwright et al., "Design of a high-sensor count fibre optic manometry catheter for in-vivo colonic diagnostics," Opt. Express 17(25), 22423-22431 (2009). 
6. P. A. Krug, R. Stolte, and R. Ulrich, "Measurement of index modulation along an optical fiber Bragg grating," Opt. Lett. 20(17), 1767$1769(1995)$

7. R. R. Maier, J. S. Barton, and J. D. Jones, "Fiber Bragg grating location by a side-scatter technique based on cladding-mode coupling," Appl. Opt. 43(16), 3310-3314 (2004).

8. W. Margulis et al., "Heat scan: a simple technique to study gratings in fibers," Opt. Lett. 18(12), 1016-1018 (1993)

9. S. Sandgren et al., "Characterisation of Bragg gratings in fibres with the heat-scan technique," Electron. Lett. 31(8), 665-666 (1995).

10. N. Roussel et al., "Measurement of index modulation along fiber Bragg gratings by side scattering and local heating techniques," Opt. Fiber Technol. 5(1), 119-132 (1999).

11. Digital Design, Inc., http://www.evolutioninkjet.com/ (18 6 2014).

12. National Instruments Corporation, http://www.ni.com/ (18 6 2014).

13. IPHT Jena, http://www.ipht-jena.de (18 6 2014).

Simon Maunder received his BSc degree in applied physics, diploma in scientific practice from the University of Technology, Sydney, in 2001. He is an experimental scientist with the Commonwealth Scientific and Industrial Research Organisation (CSIRO), Australia. He has been working on fiber optic-based sensing and labviewbased instrumentation systems used for medical device in vivo diagnostics and infrastructure monitoring applications for the past 7 years. Prior to this, he has worked on electronics and microcontroller-based instrumentation systems for aluminum industry and geophysical applications.

Neil Blenman is a senior technical officer with the Commonwealth Scientific and Industrial Research Organisation (CSIRO) Australia. $\mathrm{He}$ has been working for the past 8 years on the mechanical engineering design, manufacture, and research support for fiber optic-based sensing instrumentation systems used for medical device and infrastructure monitoring applications. Prior to this, he worked on optical systems for astronomy, plasma destruction of dangerous chemicals, wool measurement, and industrial filtration.

John Arkwright has an extensive background in optical fiber technology and devices, initially for the telecommunications industry and, more recently, for biomedical sensing. He has worked in both industrial R\&D and academic roles always with an emphasis on developing and commercialising new technologies. He has experience at many different levels from fundamental research to production line engineering. He is now working for Flinders University, building a team focused on the design, fabrication, and commercialization of fiber optic catheters for in vivo diagnostics.

Biographies of the other authors are not available. 